\title{
El Mindfulness como método para la mejora de las relaciones interpersonales
}

\section{Mindfulness as a method for improving interpersonal relationships}

Isabel Rodríguez de Medina Quevedo (isardmq@correo.ugr.es). Universidad de Granada

Fecha de recepción: 28 de mayo de 2012

Fecha de aceptación: 11 de junio de 2012

Localizador: http:www.ugr.es/local/miguelgr/ReiDoCrea-Vol.1-Art.18-Rodriguez.pdf

\section{Resumen}

Muchos de los problemas sociales se remontan a las dificultades de comunicación interpersonal. Este ensayo examina el potencial de la relación entre los estados de la mente, su anatomía y las interacciones sociales seguido por la eficacia de los programas Mindfulness para la mejora de los males sociales.

Palabras claves: relaciones interpersonales, mindfulness, emoción, neurobiología, apego.

\section{ABSTRACT}

Many social problems begin with difficulties in interpersonal communication. This essay examines the potential of the relationship between states of mind, its anatomy, and social interaction followed by the efficacy of Mindfulness programs to improve social ills.

Key words: interpersonal relationships, mindfulness, emotion, neurobiology, attachment. 


\section{INTRODUCCION}

No se puede solucionar un problema partiendo de la misma "conciencia" o perspectiva que lo provocó." Albert Einstein

En los últimos tiempos, se ha dado especial relevancia al método Mindfulness ya que es un fenómeno emergente con un gran potencial y transformador de una filosofía occidental que cada año produce un acrecimiento de malestares psicológicos.

Mindfulness es un hecho tan simple, que se refiere a algo que todos experimentamos, en mayor o en menor medida, en nuestras experiencias cotidianas. Referido a la capacidad de ser consciente de lo que pensamos, sentimos o estamos haciendo, tal y como yo estoy intentando al escribir éstas breves palabras. Podríamos decir, que Mindfulness, traducido al español como "conciencia plena" o "atención plena", es la conciencia que emerge cuando de un modo deliberado prestamos atención a las acciones del presente sin juzgarlo.

Existen dos opciones antagónicas en el sendero cognitivo, el camino derivativo de la automatización o el de la conciencia, es decir, vivir en un estado de piloto automático o vivir con atención plena (Miró, 2007). Así podemos estar presentes en "cuerpo y alma" con todos los sentidos o dejar que la automatización y el procesamiento de arribaabajo, aquel guiado por nuestras experiencias previas, sea el que viva las experiencias. Un ejemplo clarividente sería el siguiente, cuando un individuo come una fruta desconocida, como el mangostán de la India, a priori, disfruta de su sabor agridulce, del color, de una textura carnosa, principalmente saborearía esa novedad de pieza gustativa con atención y conciencia plena. Tanto la fruta como el individuo serían una unidad, se fusionan en un momento. Seguidamente, se realizaría una conexión con los esquemas cognitivos donde se asociaría con el sabor del melocotón y la frambuesa y el colorido de ésta última, a partir de éste momento se produce el susodicho procesamiento de arriba - abajo, provocando una segregación entre el alimento y la persona a través de un marco conceptual, existe una evaluación y distinción ya no se siente de forma pura, sincera, plena o atenta. Por lo tanto, Mindfulness para la mayoría de los autores y a nuestro parecer, es la acción de tomar conciencia del momento del presente, prestando atención: sin juzgar, sin evaluar y sin reaccionar a ella (Kabat-Zinn, 2003), tal y como ha sucedido con esa nueva fruta.

Una de sus mayores coyunturas es que no depende de ninguna ideología o creencia religiosa específica, ya que en ciertos casos la cultura popular lo relaciona con la tradición budista. Por lo tanto, los beneficios son accesibles a todos sin dependencia de su religión o filosofía de vida.

El enfoque Mindfulness se encuentra dentro de las terapias denominadas de tercera generación, es un hito en el desarrollo evolutivo del qué hacer terapéutico/psicológico que en estos últimos días principalmente utiliza como referente la Terapia Cognitivo Conductual (TCC), en resiliencia a que a nuestro parecer presenta ciertas limitaciones. Las características de las denominadas terapias de tercera generación (Vallejo Pareja, 2009), vendrían definidas por las siguientes: la primera es que adoptan una perspectiva más experiencial y utilizan estrategias de cambio de carácter indirecto (ampliando el abanico de aspecto concretos a determinar); la segunda, es que no se observan las formas de las conductas, es decir, el qué representan, sino más bien, las funciones de las conductas (incidiendo en que ciertas conductas actúan sobre otras). Por lo tanto, a nuestro parecer, para éste tipo de terapias lo relevante es el comportamiento en el contexto concreto en el que se presenta la conducta, centrándose mayormente en la variabilidad que en la reiteración de un determinado 
comportamiento. Interesándose más en el contexto real, o presente, que en la representación imaginaria o simbólica.

De la siguiente afirmación se puede ratificar que el Mindfulness, tenga como objeto el momento presente, para nuestros queridos lectores, vendría derivado de éste momento gratificante de lectura, sin ofrecer la importancia a la reminiscencia en el pasado o a la predicción del futuro: "¿Qué hará usted dentro de una hora? No importa solo sienta y sea consciente del instante". El cómo aprender a atender plenamente a la momentaneidad del presente reconociendo la realidad puede plantear la modificación conductual y la mejora de la fisiología cerebral (Siegel, 2011).

\section{DESARROLLO}

\section{Neurobiología interpersonal}

Siegel (2007) acuña el término neurobiología interpersonal (IPNB: Interpersonal Neurobiology) a una área interdisciplinar que tiene como objeto analizar cómo las relaciones interpersonales afectan al cerebro. Según este autor, las relaciones tanto primarias, como secundarias y las derivadas de los elementos del entorno cultural delimitarán el funcionamiento de nuestro sistema nervioso.

Por otra parte, la mayor parte de los trabajos de neuroimagen que diseminan el Mindfulness destacan una zona cerebral que destaca con superioridad a las restantes, la denominada corteza prefrontal. Esta región cerebral está involucrada en la planificación de comportamientos cognitivamente complejos, en las características de la personalidad, en los procesos de toma de decisiones complejas y en el control y conducta social idónea. La corteza prefrontal es el sustrato anatómico de las principales funciones integradores del cerebro humano y representa esa parte relevante de años de evolución del ser humano.

En lo referente a las relaciones interpersonales que es la temática que nos atañe, Siegel en su libro "The Mindful Brain" (2007), citado por V. Simón (2007) destaca nueve funciones de la corteza prefrontal medial: regulación corporal, comunicación sincronizada con otras mentes a través de procesos de resonancia, equilibrio emocional, flexibilidad de respuesta, empatía, auto-conocimiento, modulación del miedo, intuición y moralidad. Son ítems relacionados y desarrollados en las interacciones de apego seguro. Para aquellos que no conocen la terminología de la palabra apego, Bowlby (1958) expuso una serie de patrones diversos de apego que en un futuro inmediato reflejan la traducción de la experiencia interpersonal en la estructura biológica y conductual, favoreciendo el afrontamiento positivo de los conflictos interpersonales.

¿Pero qué sucede cuando el individuo no ha adquirido en las relaciones parentofiliares un apego seguro? ¿No desarrolla estos ítems? Con el tiempo... con otra tipología de interacciones... con otros miembros familiares... se podría producir ese desarrollo madurativo cerebral, sin embargo, en algunos casos existiría una deficiencia. Así pues, el estilo de apego que se adquiera en la tierna infancia sigue manifestándose en las relaciones durante la edad adulta, influyendo inclusive en las emociones. El Mindfulness puede cubrir los diversos elementos citados y provocar una conexión interna no derivada de las conductas parentales que facilite la integración de una serie de herramientas cognitivas que mejoren el funcionamiento y coherencia de las relaciones.

Es decir, Siegel (2007) propone una maravillosa teoría social que avalamos, donde la actitud de consciencia plena es como una forma de auto-relación, de sintonizarse uno 
mismo, que podría crear los mismos efectos que la de una buena sintonía interpersonal, pero ésta se realizaría a modo individual.

Por lo tanto, si se produce un cambio en el susodicho sustrato a través de una práctica prolongada de Mindfulness, surgirá un efecto mejorado en las conductas interpersonales y una calidad de las relaciones e interpretaciones que desdoblarán en el bienestar social del individuo.

\section{Mejora de las relaciones interpersonales}

¿Alguien se ha preguntado alguna vez en la vida por qué es tan complejo relacionarnos con otras personas? Una de las problemática podría derivar en no saber quienes somos nosotros mismos unido a ese "buenísmo" (placer continuado). El Mindfulness nos va ayudar a cambiar o definir la visión de quienes somos, provocando que el individuo no se vea tan aislado sino parte del mundo, favoreciendo la autoobservación y eliminando ese "yo" por el "nosotros".

En las sociedades occidentales, los antropólogos destacan que vemos a los demás como entidades separadas aunque seamos miembros de un grupo, sin embargo, en otras culturas como las africanas o asiáticas el bienestar del grupo depende de todos los miembros que lo formen, creando la identidad desde un espectro más amplio. Sin embargo, el Mindfulness nos enseña a ver cómo el problema radica en vernos a nosotros separados de los demás (Siegel, 2011). Es importante vernos identificados como aquello que denominan los biólogos, "redes ecológicas". Por ejemplo: sin padres, nosotros no existimos. Sin agua, no existe la vida. Son relaciones que son complejas porque todo está interconectado equilibradamente y es peligroso que nuestro pensamiento atribuya una existencia absoluta a algo, porque nada surge de la nada y todo tiene unos antecedentes: la bondad se crea, la empatía también, a través de la interconexiones del mundo (Kabat - Zinn, 2009).

A modo metáfora, la atención plena nos ayuda a comprobar que solo somos una diminuta perla componente del collar donde cada perla refleja en las otras y le produce un brillo especial. Es decir, es un collar nuevo, que no es una cosa, sino una nueva forma de experimentar el mundo, conectando diferentes elementos aislados, que nunca están aislados; es la forma occidental individualista de mantener una separación inexistente.

A su vez, ésta conexión es infinita, ha estado presente todo el tiempo y el brillo producido de cada perla, su reflejo, vendrá delimitado por el resto. Ese brillo es el que llamaríamos la autoestima e identidad porque el individuo vive en un grupo, y crea el sentido del "yo" continuamente, tal y como si fuera una esponja. Es decir, la identidad del individuo estará formada por unos patrones experienciales pasados y en continua definición cada minuto de su vida. Por esta razón, no es de extrañar que las personas postergadas por los padres o sus iguales, tengan una imagen inferior de sí mismo que influya en sus relaciones. Así pues, si somos conscientes de ese brillo y lo autobservamos, además de la separatividad, tendremos la capacidad de favorecer la arbitrariedad de nuestra identidad, creencias y valores, que muchas vendrían delimitadas por un reflejo incorrecto o erróneo de nosotros mismos. A su vez, se podría crear una mayor flexibilidad para relacionarnos con los demás.

Por otro lado, la práctica del Mindfulness nos ayuda a reconocer nuestras emociones y las actuaciones deliberativas. El Mindfulness hace frente al placer y al "buenismo" de la actual sociedad, nos hace conscientes de las emociones que provocan el malestar: estrés, ansiedad, depresión, infelicidad, apatía. Una serie de emociones que principalmente tienen un valor de adaptación y de las que no es recomendable la 
huída, si un individuo no tolera sus propias emociones las proyectará sobre los demás. La autobservación propia de emociones destructivas, nos hace más resistente a experimentar experiencias desagradables, mejorando la intolerancia a la incomodidad que pudiera producirse durante las relaciones.

Con técnicas de atención plena se produce una armonización interior que se relacionaría directamente con la empatía autentica generadas en las relaciones interpersonales, no la irreal derivada de ponerse en "el lugar del otro" donde se actúa como un espejo y centrándose en experiencias pasadas fundiéndose con el otro. Es decir, a través de la conciencia plena, no se funden las perspectivas, sino que favorecerá un yo observador frente al yo experimentador. Un yo observador, definido como aquel que no juzga, acepta, tiene curiosidad del otro. Es decir, estar con los demás en sus alegrías y penas pero reales. En conclusión, siguiendo a Siegel (2007, citado en V. Simón, 2007) experto en Mindfulness, "la atención plena puede favorecer las relaciones sanas entre individuos a través de una serie de mecanismos, como la empatía incrementada, el equilibrio emocional, la flexibilidad de respuestas y una predisposición mental a la aproximación".

\section{CONCLUSION}

Antes de concluir, es importante hacer clarividente el mensaje residual de éste trabajo, la esencia u objeto es mostrar cómo la atención plena (Mindfulness) es un modo de auto-observación terapéutica que facilita la conciencia de uno mismo, de cómo vivimos nuestra vidas presentes y una mejora de las relaciones interpersonales. Es decir, a través del Mindfulness nos sintonizamos y armonizamos con nosotros mismos (Siegel, 2007) y con los demás.

Por otro lado, el Mindfulness es una fuente de identidad, de interconexión con el mundo, de consciencia de las emociones, que favorece una serie de mecanismos y un "yo observador" que facilita la afrontación y la predisposición de las relaciones interpersonales.

\section{BIBLIOGRAFIA}

Bowlby, J. (1958). The nature of the child's tie to his mother. Internacional Journal of Psychoanalysis, 39, 350-373

Corteza prefrontal. (2012, 8 de mayo). Wikipedia, La enciclopedia libre. Fecha de consulta, junio 05, 2012.

http://es.wikipedia.org/w/index.php?title=Corteza_prefrontal\&oldid=56008215

De la Fuente, M., Franco, C., \& Salvador, M. (2010). Efectos de un programa de meditación (Mindfulness) en la medida de la alexitimia y las habilidades sociales. Psicothema, 22(3), 369-375.

Gemer, C. K. (2011). El poder del mindfulness: libérate de los pensamientos y emociones autodesctructivas. Madrid: Paidós.

Goleman, D. (1996). Inteligencia Emocional. Barcelona: Kairos

Kabat-Zinn, J. (2003). Mindfulness-based intervention in context: past, present, and future. Clinical Psychology, Science and Practice, 10, 144-156.

Kabat-Zinn, J. (2009). Mindfulness en la vida cotidiana, donde quieras que vayas, ahí estás. Barcelona: Paidós. 
Miró, M. T. (2007). La atención plena (Mindfulness) como intervención clínica para aliviar el sufrimiento y mejorar la convivencia. Revista de Psicoterapia, 17, 31-76.

Siegel, D.J. (2007). The mindful brain: Reflection and attunement in the cultivation of wellbeing. Nueva York: Norton.

Siegel, D.J. (2011). La solución Mindfulnes: prácticas cotidianas para problemas cotidianos. Bilbao: Edesclee.

Simón, V. (2007). Mindfulness y neurobiología. Revista de Psicoterapia, 66/67, 5-30.

Vallejo, M.A. (2006). Mindfulness. Papeles del Psicólogo, 27(2), 92-99.

Vallejo, M.A. (2007). El Mindfulness y la «tercera generación de terapias psicológicas». Infocop, 33, 16-18. 